Journal of Innovative Image Processing (JIIP) (2020)

Vol.02/ No. 01

Pages: $44-54$

https://www.irojournals.com/iroiip/

DOI: https://doi.org/10.36548/jiip.2020.1.005

\title{
Analysis of Artificial Intelligence based Image Classification Techniques
}

\author{
Dr. Subarna Shakya \\ Professor, Department of Electronics and Computer Engineering, \\ Central Campus, Institute of Engineering, Pulchowk, \\ Tribhuvan University. \\ Email: drss@ioe.edu.np.
}

\begin{abstract}
Time is an essential resource for everyone wants to save in their life. The development of technology inventions made this possible up to certain limit. Due to life style changes people are purchasing most of their needs on a single shop called super market. As the purchasing item numbers are huge, it consumes lot of time for billing process. The existing billing systems made with bar code reading were able to read the details of certain manufacturing items only. The vegetables and fruits are not coming with a bar code most of the time. Sometimes the seller has to weight the items for fixing barcode before the billing process or the biller has to type the item name manually for billing process. This makes the work double and consumes lot of time. The proposed artificial intelligence based image classification system identifies the vegetables and fruits by seeing through a camera for fast billing process. The proposed system is validated with its accuracy over the existing classifiers Support Vector Machine (SVM), K-Nearest Neighbor (KNN), Random Forest (RF) and Discriminant Analysis (DA).
\end{abstract}

Keywords: Fruit image classification, Automatic billing system, Image classifiers, Computer vision recognition.

\section{Introduction}

Artificial intelligences are given to a machine to work on its task independently without need of any manual guidance. All the necessary guidance and directions to be given to the machine or system are programmed once at the time of installation. This saves lot of time and energy in the working environment. A monitoring element can be employed for verifying the operations of the independent system to avoid immoral works. Adding the artificial intelligence based work to the existing automation works gives a trendy output to the human convenience. Most of the existing automations requires a human guidance at regular interval to make up the operations. The artificial intelligence based systems are generally a one time programing automation system added with human intelligence to take decisions in critical circumstances. Now adding visual intelligence to the computer makes the system to work independently without manual interruption or decision. The proposed artificial intelligence based visual system makes an independent billing process for fruits by classifying it through a camera and an algorithm. The figure 1 shows the basic structure of artificial intelligence based computer vision.

ISSN: 2582- 4252 (online) 


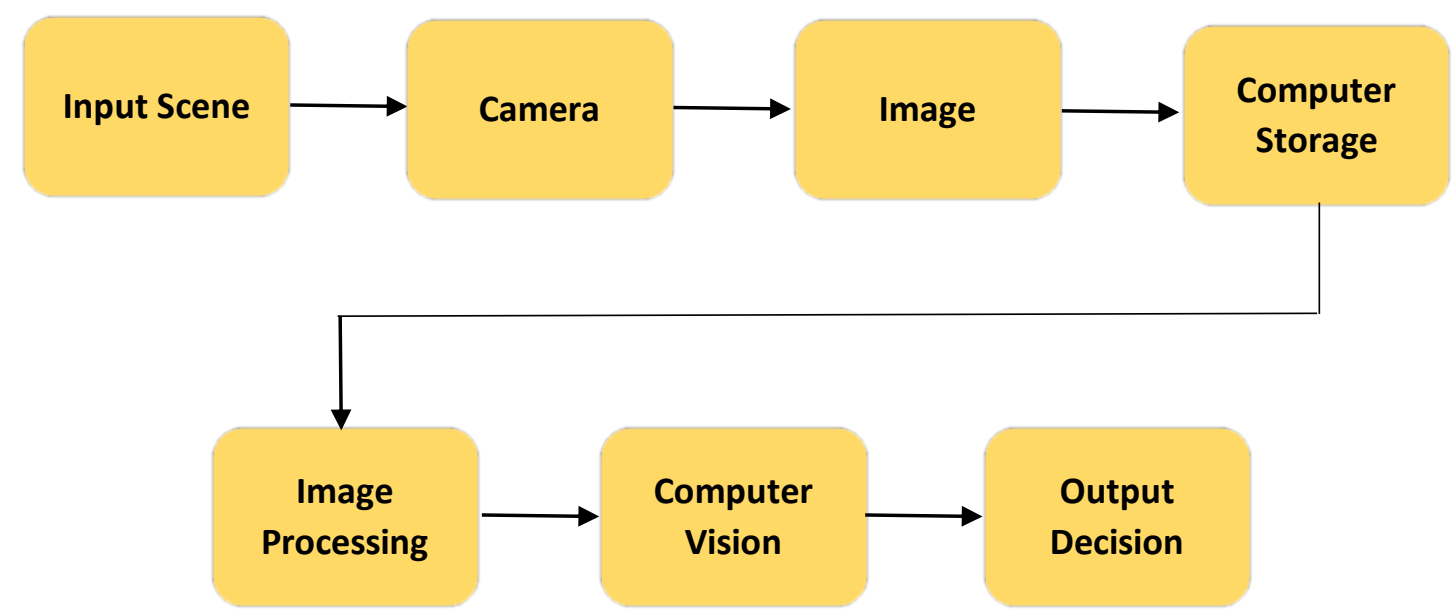

Figure 1. Basic architecture of computer vision system.

The computer vision intelligence is enforced to a system by fixing a camera to produce a visual image which can be readable by an algorithm programmed in the system. The input scene can be any kind of image, which can be taken directly with a camera or scanner. Apart from that smartphone camera and screen shots of the computer system are also applied as an image scene for computer vision system [1]. Several scanning and imaging methods are empowered in medical computer vision for diagnosing several diseases. The computer vision based diagnosing systems performs very fast and accurate. The diagnosis reports are helpful for surgical arrangement and post-surgical health estimation [2]. Apart from medical diagnosis the human body parts are captured for several applications, biometric recognition is one among such application which are helpful in authentication of a human [3]. The computer vision experiments are applied to several kind of navigation purpose with the help of pre-installed map and a physical location identification by a camera image instead of GPS system. An autonomous navigation system was experimented with an underwater vehicle for its self-navigation by a real-time image through a single camera and then the image is enhanced with a filtering technique for an effective visualization [4]. Figure 2 explores the broad application areas where the computer vision is already applied. 
Journal of Innovative Image Processing (JIIP) (2020)

Vol.02/ No. 01

Pages: 44-54

https://www.irojournals.com/iroiip/

DOI: https://doi.org/10.36548/jiip.2020.1.005

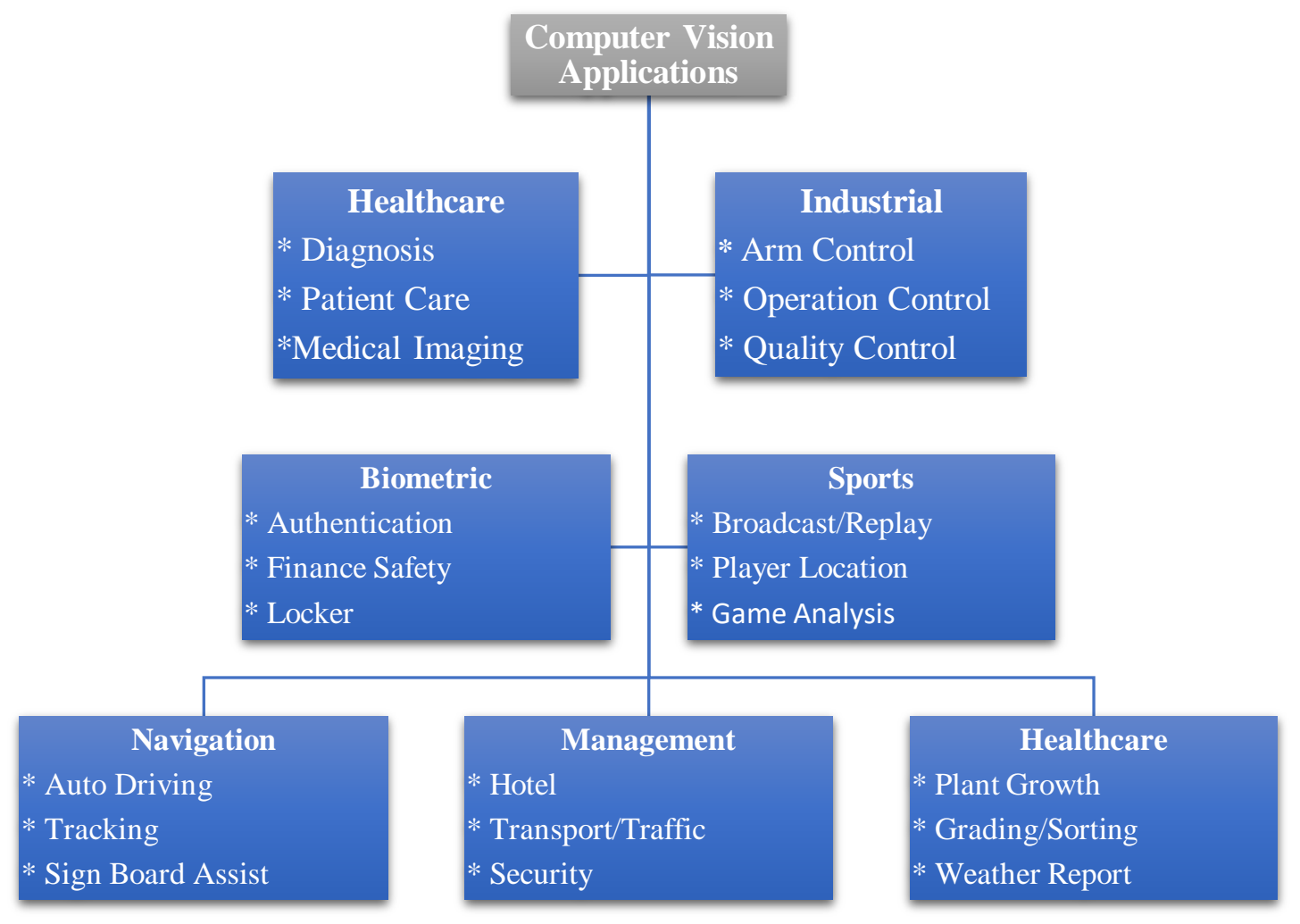

Figure 2. Existing computer vision applications

An image classification technique for automobile air-conditioning leakage detection [5] was proposed to identify the faulty portion in the circuit with the help of mean weight least square SVM algorithm. Here the captured image is pre-processed using a Bayesian filter for noise removal and type 2 adaptive system for enhancement. Apart from that a genetic algorithm was added to the enhanced image for feature selection process to train the classifiers. Labeling of images is an expensive work in the process of image classification. Labeling consumes more time and energy, but it is necessary to label the images for training process in classification techniques. A multiple instance learning [6] is a labeling technique does the process by carrying the trained image variations in to a set of bags or folders for ease of work. This technique was applied to classify the breast tumor images and the results are satisfied when comparing with the existing single instance learning technique. A neural network based technical analysis [7] was performed to a breast cancer dataset to identify the present stage of the tumor or cancer. The existing neural network based techniques are implemented here with the same dataset for its performance evaluation and the result indicates that the convolution neural network does a better job in classification when comparing with the feed forward neural network and recurrent neural network. 
Journal of Innovative Image Processing (JIIP) (2020)

Vol.02/ No. 01

Pages: 44-54

https://www.irojournals.com/iroiip/

DOI: https://doi.org/10.36548/jiip.2020.1.005

A deep learning method was performed to classify the type of vehicles [8] to be a part in intelligent traffic system. The faster region convolution neural network technique was enforced to perform the classification tasks. The proposed deep learning technique performs with higher accuracy in lesser time to classify cars and trucks images available in a public dataset. The technique is also implemented with a real time embedded processor to verify its real time performance and the processor detected an image in 0.354 seconds with acceptable accuracy. A traffic sign sensing technique [9] was structured based on computer vision technique to develop a smart transport system. The technique is helpful in path pre-planning and safe driving of a vehicle when it is manual or autonomous. The technique was framed with capsule network technology for giving better accuracy rate over convolution neural network and recurrent neural network. A segmentation process with Hough transform was added before the vision technique for image enhancement. The Naïve Bayes classifier was utilized to classify the fruit and vegetable images. The proposed technique was automatized with a texture based approach [10] for image segmentation. The images are collected for all the 360 degree angles to train the classifier for its efficient output. The feature vector for the classifier is framed by combining fractal dimension and the matrix based features extracted from the gray level image.

A semantic segmentation process was designed with cascaded convolution neural network [11] to perform in biomedical images. The designed technique is operated with an encoder and decoder for down sampling the input images to make a feature mapping in effective manner. Then the up sampling is taken into account during diagnosing time. An ImageNet pre-trained neural network [12] was approached to classify the fruit and vegetable images. The approach was tested with the customized images captured in realistic places and it has gone through with a data compression layer to detect the shape and color of the fruit and vegetables. A combination of image processing and computer vision technique [13] was implemented to predict the growth of a fruit in farming field. The growth stages are identified by changes in color of the fruit, and the changes are verified by analyzing the RGBD of an image.

A fruit classification deep learning framework [14] was proposed to detect the kind of fruits for tagging and weighing purpose. A network model of six convolution neural network is taken as first model in the algorithm and a fine-tuned geometry learning model is entitled as second model of the algorithm. A public dataset along with a personal dataset is used here to understand the performance of the proposed framework and it achieves 85 to 99 percentage of accuracy in non-clear image and clear full image. The computer vision based applications equipped with machine learning and deep learning techniques were reviewed [15] and found the computer vision based technology is a reliable method for visual perception in any scheme or applications. A neural network based algorithm with good architecture can predict any object by a digital camera or an imaged already kept inside a drive of a computer to analyze. The challenges in detection and grading of fruits and vegetables system were reviewed [16] and concluded as, the computer vision technologies may reduce the time for fruits/vegetables deduction but the technology may reduce the grading efficiency of the process. 
Journal of Innovative Image Processing (JIIP) (2020)

Vol.02/ No. 01

Pages: $44-54$

https://www.irojournals.com/iroiip/

DOI: https://doi.org/10.36548/jiip.2020.1.005

\section{Proposed Method}

The proposed artificial intelligence based image classification technique performance is analyzed with several image classifiers for its better outcome. The images which are carried for the work is download from kaggle image detection database consists of 240 images in training folder with group of apple, banana and orange images. Similarly there are 60 images in test folders. Figure 3. indicates few sample images used for training.

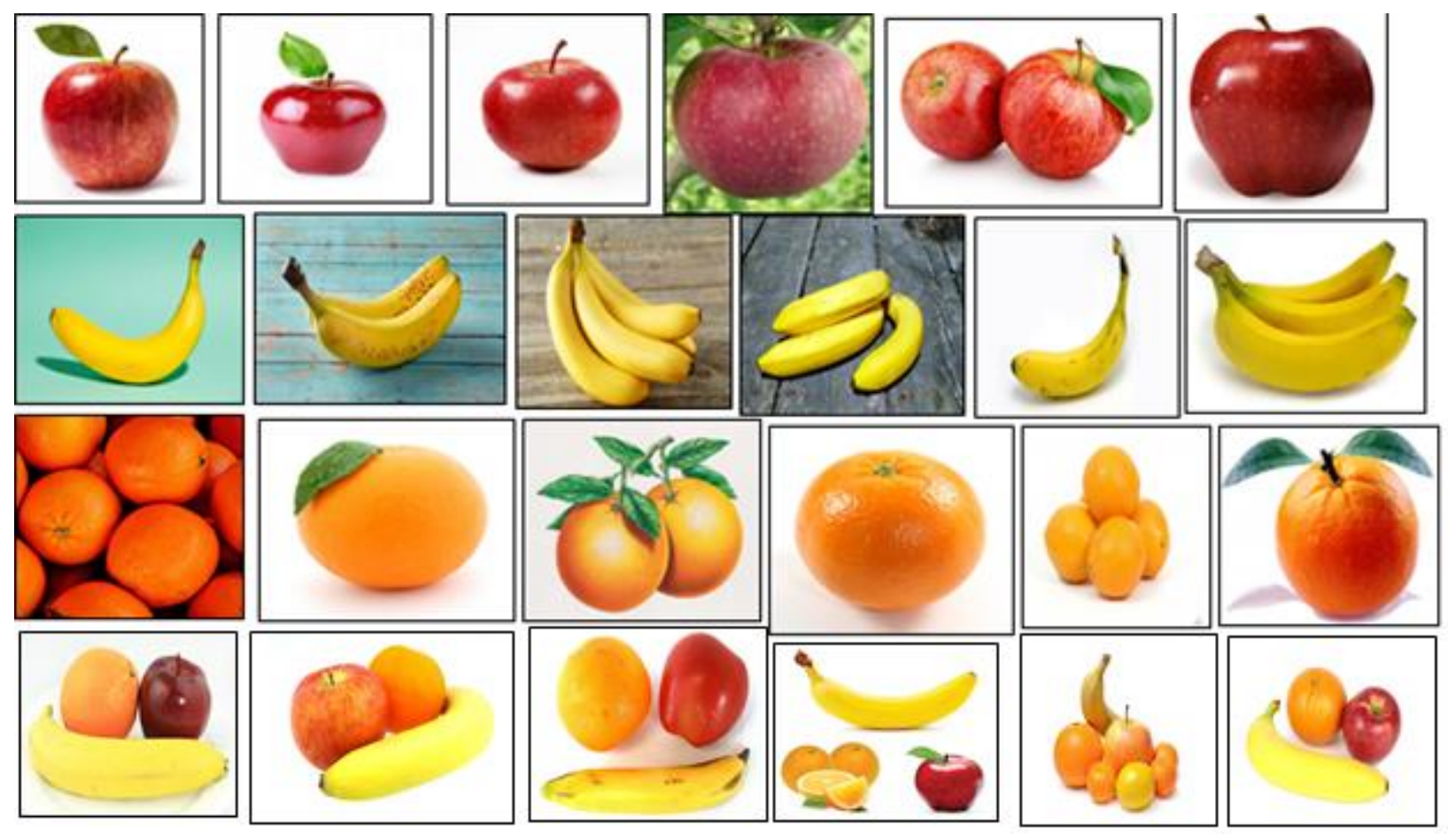

Figure 3. Sample dataset images.

The proposed method classifies the fruit by detecting its color, shape and size. Figure 4 specifies an overview of the proposed classification system. Before carrying the images for classification, the proposed system improvise the visual rating of the images by preprocessing it. The image features are extracted from the preprocessed image and then the extracted features are merged with the trained features for comparison during classification. 


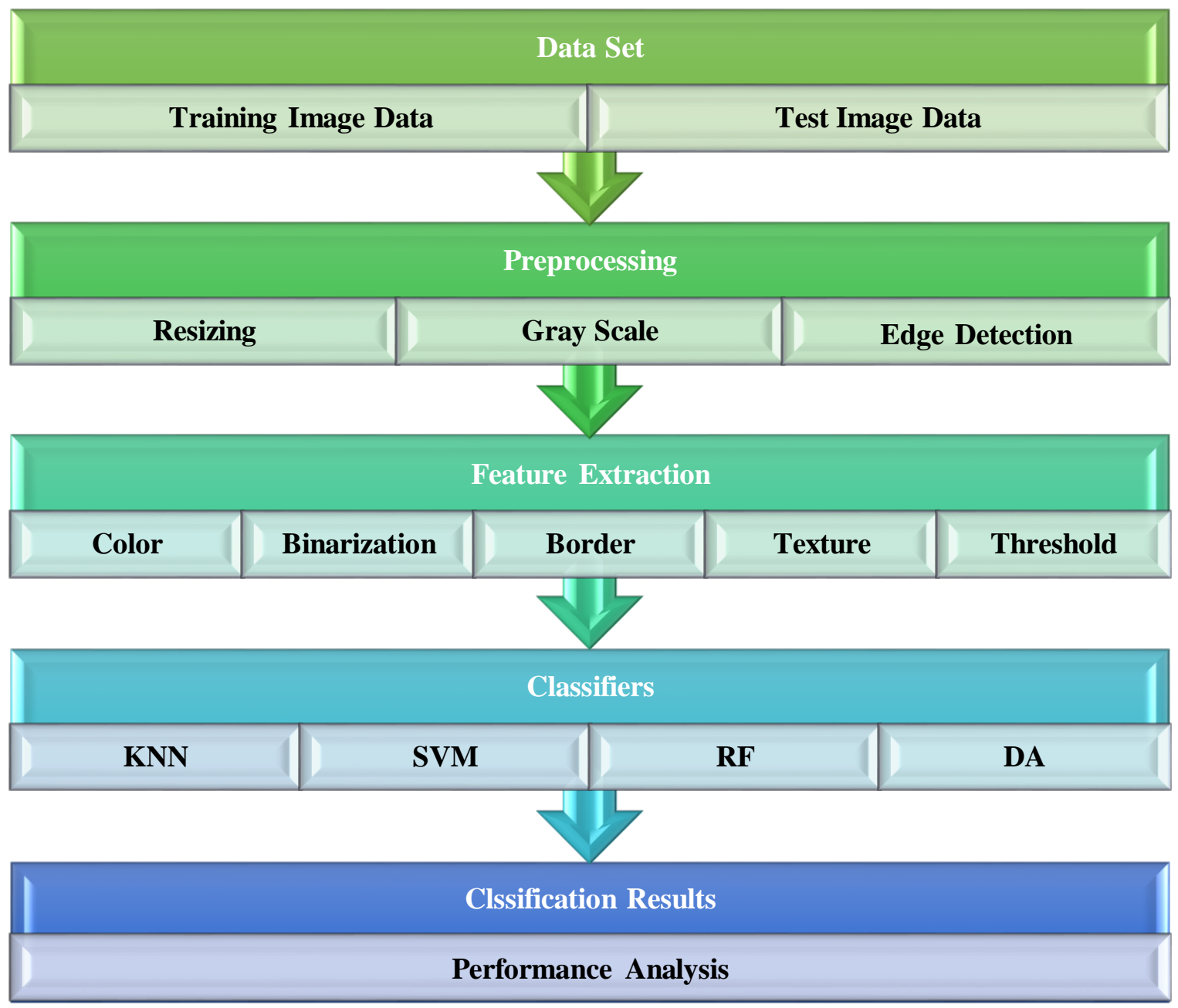

\section{Figure 4. Overview of proposed system.}

The image preprocessing of the proposed system consists of image resizing, gray scale conversion and edge detection for extracting its features in an efficient manner. The test and training images are first resized to $\mathrm{a}^{*} \mathrm{~b}$ pixels to follow a general cropping ratio. For better performance in edge detection, the images are converted into gray scale image using the general summing format as indicated in equation 1.

$\alpha=[(a * b)(R * 0.2989+G * 0.587+B * 0.114)]$

Eqn. 1

The gray scaled image $(\alpha)$ is moved for edge detection with convolution to Gaussian filter coefficient and canny filter operation for vector orientation. The gradient vector is estimated and with nonmaximum suppression matrix followed by threshold strategy. By using this the weaken edges of image $\alpha$ 
Journal of Innovative Image Processing (JIIP) (2020)

Vol.02/ No. 01

Pages: 44-54

https://www.irojournals.com/iroiip/

DOI: https://doi.org/10.36548/jiip.2020.1.005

is connected towards the bright edges of the image. This avoids the filter to detect noise as edges. The threshold of the image $\alpha$ is calculated from the edge preserved image and it is carried for background blocking using dilation tool as expressed in equation 2 . The dilated image $\beta$ is further made to binary image.

$\beta=\max \left\{A\left(a-a^{\prime}, b-b^{\prime}\right) \downarrow\left(a^{\prime}, b^{\prime}\right) \in D_{B}\right\}$

Eqn. 2

The color feature of an image is extracted by its mean RGB value after cropping it to binary image value for easy identification of image regions. The image color is analyzed by cropping several portions in an image on various areas to calculate the summation and mean of the color presence in the image. The cropped portions in the images are uniform for all the images to make it reliable and uniform. From the binary image $\gamma$, the area feature $\mu$ of the fruit is extracted. The shape of the binary image is extracted by finding its roundness, and diameter. The extracted features from the image is taken in to the classifiers for training the algorithm architecture. All the classifiers performs on its own characteristics, the performance difference of the classifiers are happening due to its nature of alignment. A combination of perfect preprocessing techniques with extract features is not only enough for classification. Identification of right classifiers for the proposed image is necessary to make a better computer vision structure.

The SVM classifier classifies the images with the help of binary acyclic graph and we can make an option to compare the extracted graph values to compare with the available image combinations or with some particular combination. The KNN classifier classifies an image by comparing the ' $\mathrm{K}$ ' value of the training data with test data for finding closeness with the testing image or data. The ' $\mathrm{K}$ ' values are estimated from the feature extraction carried out during the training process. Euclidean equation principle is employed in KNN classifier for identifying the similarity. The vector samples are randomly collected in RF classifiers and it assumes the training images as trees and is features as its branches. The trees of group form a combination to matchup their properties with the test images. The DA classifies the image based on the Gaussian distribution variations formed inside the classifier and it tries to predict the test data in with the smallest matched to the Gaussian distributed value.

\section{Results and Discussion}

The classifiers performance are analyzed with the kaggle fruit image detection dataset in MATLAB platform with i7 processor of 8GB RAM. Table 1 specifies the confusion matrix for the SVM classifier. The performance of the classifiers are analyzed with 58 test images in combination of 18 apples, 17 bananas and 18 oranges along with that 5 numbers of mixed fruit images are given to the classifiers for detecting its specificity and negative predictive value. Table 2, 3 and 4 indicates the confusion matrix of KNN, RF and DA classifiers respectively. Table 5 point outs the performance metric analysis of the classifiers in terms 
Journal of Innovative Image Processing (JIIP) (2020)

Vol.02/ No. 01

Pages: 44-54

https://www.irojournals.com/iroiip/

DOI: https://doi.org/10.36548/jiip.2020.1.005

of accuracy, sensitivity, specificity, Positive Predictive Value (PPV) and Negative Predictive Value (NPV). Similarly, figure 5 exhibits the graphical performance comparison of classifiers.

Table 1. Confusion matrix of SVM classifier.

\begin{tabular}{|c|c|c|c|c|c|c|}
\hline Status & \multicolumn{6}{|c|}{ Predicted } \\
\hline \multirow{6}{*}{ Actual } & Fruits & Apple & Banana & Orange & Mixed & Sum \\
\hline & Apple & 14 & & 1 & 3 & 18 \\
\hline & Banana & & 13 & & 4 & 17 \\
\hline & Orange & 1 & & 15 & 2 & 18 \\
\hline & Mixed & 1 & 2 & & 2 & 5 \\
\hline & Sum & 16 & 15 & 16 & 11 & 58 \\
\hline
\end{tabular}

Table 2. Confusion matrix of KNN classifier.

Status Predicted

\begin{tabular}{|c|c|c|c|c|c|c|}
\hline \multirow{2}{*}{. } & \\
\hline & Fruits & Apple & Banana & Orange & Mixed & Sum \\
\hline \multirow{5}{*}{ Actual } & Apple & 17 & & & 1 & 18 \\
\hline & Banana & & 16 & & 1 & 17 \\
\hline & Orange & & & 17 & 1 & 18 \\
\hline & Mixed & & & 1 & 4 & 5 \\
\hline & Sum & 18 & 16 & 17 & 7 & 58 \\
\hline
\end{tabular}

Table 3. Confusion matrix of RF classifier.

\begin{tabular}{|c|c|c|c|c|c|c|}
\hline Status & \multicolumn{6}{|c|}{ Predicted } \\
\hline \multirow{6}{*}{ Actual } & Fruits & Apple & Banana & Orange & Mixed & Sum \\
\hline & Apple & 14 & & 2 & 2 & 18 \\
\hline & Banana & & 12 & & 5 & 17 \\
\hline & Orange & 3 & & 12 & 3 & 18 \\
\hline & Mixed & 1 & 2 & 1 & 1 & 5 \\
\hline & Sum & 18 & 14 & 15 & 11 & 58 \\
\hline
\end{tabular}

Table 4. Confusion matrix of DA classifier.

\begin{tabular}{cl|ccc|c|c}
\hline \multirow{2}{*}{ Status } & \multicolumn{5}{c}{ Predicted } & \\
\hline \multirow{4}{*}{ Actual } & Fruits & Apple & Banana & Orange & Mixed & Sum \\
\cline { 2 - 7 } & Apple & 17 & & 1 & 18 \\
\cline { 2 - 7 } & Banana & & 15 & 2 & 17 \\
\cline { 2 - 7 } & Orange & 2 & 15 & 1 & 18 \\
\hline
\end{tabular}


Journal of Innovative Image Processing (JIIP) (2020)

Vol.02/ No. 01

Pages: 44-54

https://www.irojournals.com/iroiip/

DOI: https://doi.org/10.36548/jiip.2020.1.005

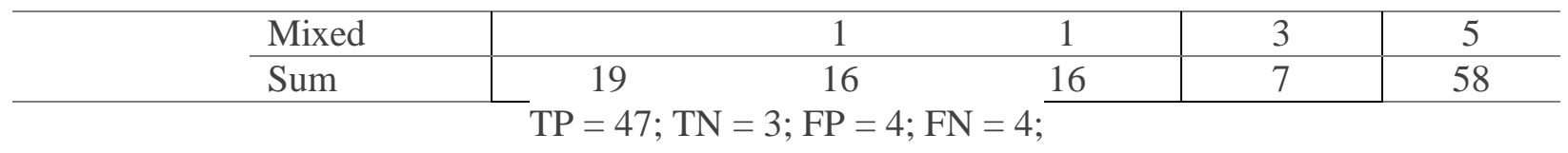

Table 5. Performance analysis of classifiers.

\begin{tabular}{c|ccccc}
\hline Algorithm & Accuracy & Sensitivity & Specificity & PPV & NPV \\
\hline SVM & 75.862 & 82.352 & 28.571 & 89.317 & 18.182 \\
\hline KNN & 93.103 & 94.339 & 80 & 98.039 & 57.142 \\
\hline RF & 67.241 & 79.166 & 10 & 80.851 & 9.091 \\
\hline DA & 86.207 & 92.156 & 42.857 & 92.156 & 42.857 \\
\hline
\end{tabular}

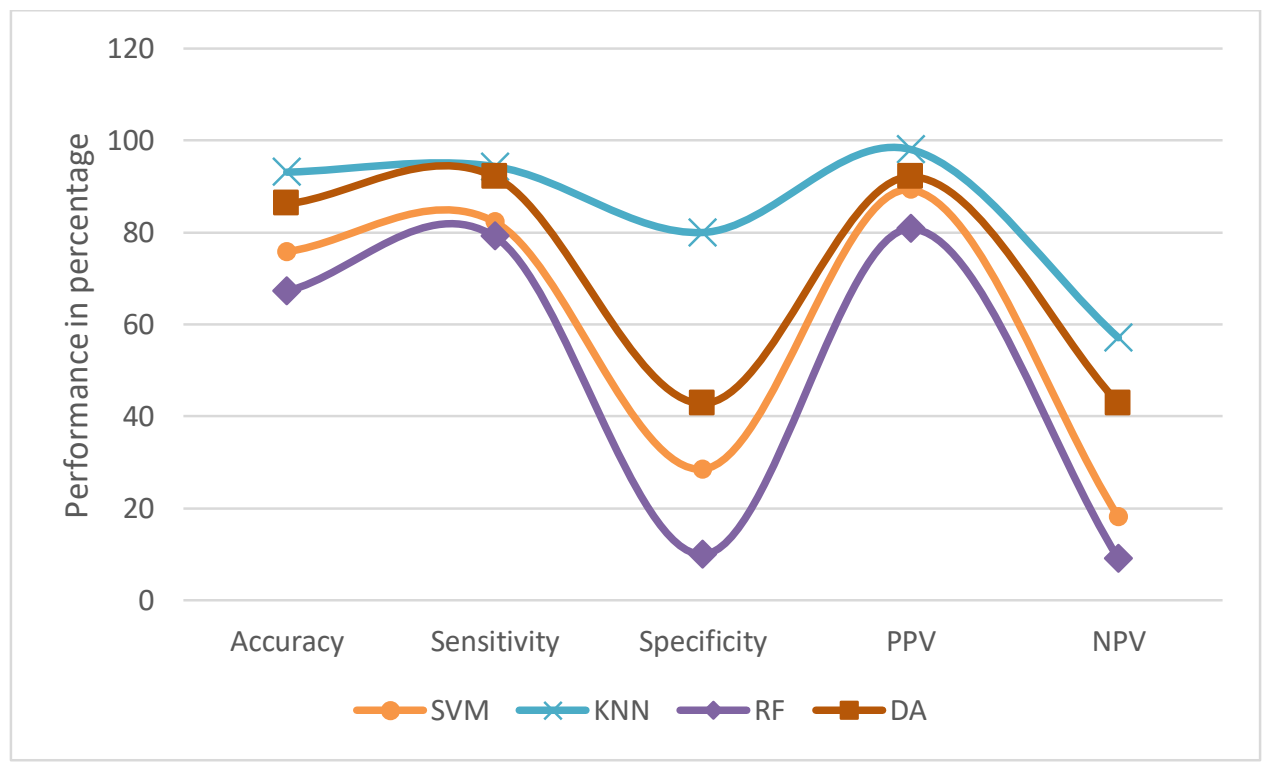

Figure 5. Performance comparison of classifiers.

From the analysis table and comparison chart the KNN classifier classifies the images with better accuracy of $93.103 \%$ which is better than all the other classifiers. The RF classifier performance is comparatively lesser than the other classifiers with $67.241 \%$. The DA classifier and SVM classifiers are comes in between the performance of KNN and RF classifier. The accuracy, sensitivity and PPV performance are slightly differ to each classifiers but the specificity and NPV issues a larger difference value when comparing with KNN. This happens because of untrained mixed fruit category to the classifiers. Even though without the training data of mixed fruits the KNN was able to separate those images as not classified images, instead all the other classifiers were actively made it to point it as on other classification categories. The performance of specificity and NPV can be improved when the mixed category images are trained with the classifiers but at the same time the performance of KNN may also get increase among all the another classifiers.

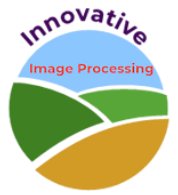


Journal of Innovative Image Processing (JIIP) (2020)

Vol.02/ No. 01

Pages: $44-54$

https://www.irojournals.com/iroiip/

DOI: https://doi.org/10.36548/jiip.2020.1.005

\section{Conclusion}

The proposed artificial intelligence based image classification system suggests a preprocessing method with KNN classifier can classify the fruit images effectively than the other classifiers with accuracy of $93.103 \%$. The proposed methodology can be merged with a weighing machine for faster billing process. It can be achieved with a sample fruit to be kept under camera before weighing all the fruits, from the camera the image can be forwarded to the computer storage for algorithm performance to classification. The proposed algorithm has to be improved in specificity and NPV to meet the real time challenges and the mixed fruit evaluation has to be improved for real time performance evaluation. This can be achieved by training the network model with more number of mixed fruit images.

\section{References}

[1] Mohamed, Ashraf A., and Ahmed A. Shalaby. "Digital imaging devices as sensors for iron determination." Food chemistry 274 (2019): 360-367.

[2] Hezekiah, James DK, and Shanty Chacko. "A Review on Cornea Imaging and Processing Techniques." Current Medical Imaging 16, no. 3 (2020): 181-192.

[3] Jacob, I. Jeena. "Capsule Network Based Biometric Recognition System." Journal of Artificial Intelligence 1, no. 02 (2019): 83-94.

[4] Manzanilla, Adrian, Sergio Reyes, Miguel Garcia, Diego Mercado, and Rogelio Lozano. "Autonomous Navigation for Unmanned Underwater Vehicles: Real-Time Experiments Using Computer Vision." IEEE Robotics and Automation Letters 4, no. 2 (2019): 1351-1356.

[5] Manoharan, Samuel. "Image detection, classification and recognition for leak detection in automobiles." Journal of Innovative Image Processing (JIIP) 1, no. 02 (2019): 61-70.

[6] Sudharshan, P. J., Caroline Petitjean, Fabio Spanhol, Luiz Eduardo Oliveira, Laurent Heutte, and Paul Honeine. "Multiple instance learning for histopathological breast cancer image classification." Expert Systems with Applications 117 (2019): 103-111.

[7] Vijayakumar, T. "Neural network analysis for tumor investigation and cancer prediction." Journal of Electronics 1, no. 02 (2019): 89-98.

[8] Wang, Xinchen, Weiwei Zhang, Xuncheng Wu, Lingyun Xiao, Yubin Qian, and Zhi Fang. "Real-time vehicle type classification with deep convolutional neural networks." Journal of Real-Time Image Processing 16, no. 1 (2019): 5-14.

[9] Koresh, M. H., and J. Deva. "Computer vision based traffic sign sensing for smart transport." $J$. Innovative Image Process.(JIIP) 1, no. 01 (2019): 11-19.

[10] Jana, Susovan, Ranjan Parekh, and Bijan Sarkar. "Automatic Classification of Fruits and Vegetables: A Texture-Based Approach." In Algorithms in Machine Learning Paradigms, pp. 71-89. Springer, Singapore, 2020.

[11] Bindhu, V. "Biomedical image analysis using semantic segmentation." Journal of Innovative Image Processing (JIIP) 1, no. 02 (2019): 91-101. 
Journal of Innovative Image Processing (JIIP) (2020)

Vol.02/ No. 01

Pages: $44-54$

https://www.irojournals.com/iroiip/

DOI: https://doi.org/10.36548/jiip.2020.1.005

[12] Steinbrener, Jan, Konstantin Posch, and Raimund Leitner. "Hyperspectral fruit and vegetable classification using convolutional neural networks." Computers and Electronics in Agriculture 162 (2019): 364-372.

[13] Chandy, Abraham. "RGBD analysis for finding the different stages of maturity of fruits in farming." Journal of Innovative Image Processing (JIIP) 1, no. 02 (2019): 111-121.

[14] Hossain, M. Shamim, Muneer Al-Hammadi, and Ghulam Muhammad. "Automatic fruit classification using deep learning for industrial applications." IEEE Transactions on Industrial Informatics 15, no. 2 (2018): 1027-1034.

[15] Suma, V. "Computer vision for human-machine interaction-review." Journal of trends in Computer Science and Smart technology (TCSST) 1, no. 02 (2019): 131-139.

[16] Zhang, Baohua, Baoxing Gu, Guangzhao Tian, Jun Zhou, Jichao Huang, and Yingjun Xiong. "Challenges and solutions of optical-based nondestructive quality inspection for robotic fruit and vegetable grading systems: A technical review." Trends in food science \& technology 81 (2018): 213-231.

ISSN: 2582- 4252 (online) 